\title{
Investigation on the Microbiological and Biochemical Properties of Kimchi in the Solid-state Model System Designed for Fermented Sausages
}

\author{
Joo-Yeon Lee* \\ Korea Livestock Products HACCP Accrediation Service, Anyang 430-731, Korea
}

\begin{abstract}
The objective of this study was to investigate the potential of the application of kimchi LAB as starter culture in the production of fermented sausages. For this, the solid-state model media composed to simulate the substantial conditions of meat mixtures were fermented for $120 \mathrm{~h}$ after the treatment with different concentrations of kimchi $(0.5,1.0,1.5,3.0$, and 5.0\%) and lyophilized kimchi-powder (0.2\% and 0.5\%). During the fermentation period, the growth of total viable cells and LAB, and the changes of $\mathrm{pH}$ and titratable acidity were investigated. The initial LAB counts ranged from 7.18 to $8.34 \mathrm{Log}$ CFU/ $\mathrm{mL}$ for kimchi media and from 6.93 to $6.94 \mathrm{Log}$ CFU/mL for kimchi-powder media depending on the added concentrations. The kimchi LAB in this study were not influenced by the immobilized condition for their adaptation and growth by showing no lag phase and thus acted similar as in the submerged medium. The initially increased counts reached around 9 Log CFU/ $\mathrm{mL}$ in $12 \mathrm{~h}$ independent of the concentrations of added kimchi. However, the growth and metabolic activity of kimchi-powder LAB were influenced by the immobilized condition. Supposedly, as the nutrient supply in solid-state depended solely on diffusion, these differences in the souring properties were caused by the LAB topography in the medium matrix. Nevertheless, the differences in the numbers of LAB between two media were less than 0.5 Log units and the pH drop in the solidstate batches was quite rapid and reached low values. Therefore, it can be assumed that kimchi and kimchi-powder LAB showed the utility as the substitute of commercial starter culture even without a rehydrating pretreatment.
\end{abstract}

Key words: Fermented sausage, solid-state fermentation, kimchi, kimchi-powder, lactic acid bacteria, starter culture

\section{Introduction}

At the area of fermented sausage production, the requirements of large-scale, low-cost industrial production with short ripening times and highly standardized end-products has made research intensive, when the first systematic studies on the microbiology and the production of fermented sausage were published (Kroeckel, 1995). Since then, the interest in starter cultures concerning meat fermentation has greatly increased, and numerous excellent reviews deal with the history and the physiological and technological aspects of starter cultures (Bacus and Brown, 1981; Geisen et al., 1992; Kunz, 1994; Luecke and Hechelmann, 1986; Smith and Palumbo, 1983). Among the microbial components of meat starter cultures that are commercially available, the application of lactic acid bacteria (LAB) as starter culture is ubiqui-

\footnotetext{
*Corresponding author : Joo-Yeon Lee, Korea Livestock Products HACCP Accreditation Service, Anyang 430-731, Korea. Tel: 82-31-390-5246, Fax: 82-31-465-6698, E-mail: ljy@ihaccp. or.kr
}

tous due to their technological and hygienic advantages compared to "natural fermentation" that is governed by the microflora derived from the raw materials or during processing (Hammes and Knauf, 1994; Kunz 1989; Roca and Incze, 1990). The beneficial property of LAB to decrease $\mathrm{pH}$ resulted from sugar utilization and production of organic acids (mainly lactic acid) is essential for the desired achievement of texture, color, flavor, and above all hygienic safety in sausages (Kunz, 1989).

The most used and commercially available LAB as starter culture in the production of fermented sausages are Lactobacillus curvatus, L. sake, L. plantarum, and Pediococcus acidilactici, since they are highly competitive in and well adapted to the environment of fermented sausages. These species of LAB are also the common ones that emerge in kimchi and continuously participate in its fermentation. Indeed, as shown in the previous study, $L$. brevis, L. sake, L. plantarum, L. curvatus, and Leuconostoc mes.mes./dent were the main LAB isolated and identified from kimchi (Lee et al., 2006). Although the environmental conditions in kimchi and fermenting sausages are different, the LAB emerged in kimchi during 
fermentation at $20-30^{\circ} \mathrm{C}$ are expected to have the potential utility as a substitute of starter culture in the production of fermented sausages (Hwang et al., 1960; Lee et al., 2006).

The utility potential of kimchi LAB as starter culture in the production of fermented sausages was investigated at the previous study using submerged model-medium, in which the conditions were composed to simulate those of meat mixture (Lee and Kunz, 2009). A real sausage mixture, however, has different features from the submerged medium. The nutrients are not distributed evenly and the mobility of microorganisms is limited in sausage mixtures due to insufficient water content as compared to the liquid medium. Microorganisms cannot be evenly distributed and their growth can only take place in small groups, so-called "nests". The nests are usually enclosed in small "cavities" within the sausage mixture. As the fermentation progresses, bacteria are captured in these "cavities" and they are immobilized there. Thus, sausage ripening can be regarded as 'solid-state fermentation' (Katsaras and Leistner, 1988). In the beginning of the fermentation, the nutrients are distributed almost homogeneously in mixed media. In a solid-state mixture, bacteria start to consume nutrients from their surroundings. As the fermentation progress, continues a depletion of nutrients in their surroundings develops. In such a condition, the nutrients are supplied to the bacteria via diffusion. As a result, diffusion is a decisive factor in the determination of fermentation velocity.

In this study, the microbiological and biochemical properties of LAB originated from kimchi under the fermented sausage condition designed as a fixed phase ("solidstate") were investigated to verify the utility potential of kimchi LAB as starter culture for the production of fermented sausages. The type of kimchi used was verified as fresh one as well as lyophilized kimchi-powder.

\section{Materials and Methods}

\section{Preparation of kimchi and kimchi-powder}

The preparation of kimchi was carried out based on the "baechu-kimchi" recipe. The ingredients are listed in Table 1. Previously, garlic, ginger, and leek were chopped. Paprika, purchased from Korea, and sugar were weighed. The Chinese cabbage was cut into pieces of $3 \times 3 \mathrm{~cm}$ in length and soaked in $15 \%(\mathrm{w} / \mathrm{v})$ brine for $30 \mathrm{~min}$. The soaked cabbage was washed twice with fresh water and then drained for $30 \mathrm{~min}$. The prepared ingredients were mixed well and then distributed evenly on the Chinese
Table 1. Composition of kimchi materials

\begin{tabular}{lc}
\hline \hline Materials and Ingredients & Percentage share (\%) \\
\hline Salted Chinese cabbage & 90 \\
Leek & 4 \\
Paprika powder (Korean) & 2 \\
Garlic & 2 \\
Ginger & 1 \\
Sugar & 1 \\
\hline
\end{tabular}

cabbage. The kimchi mixture was put into a polyethylene bag (iUL, Spain) and closed under vacuum. It was fermented at $20^{\circ} \mathrm{C}$ for $6 \mathrm{~d}$, respectively. For the production of kimchi-powder, fermented kimchi (at $20^{\circ} \mathrm{C}$ for $5 \mathrm{~d}$ and at $7^{\circ} \mathrm{C}$ for $10 \mathrm{~d}$ ) was frozen in a round flask at $-72^{\circ} \mathrm{C}$ and later dried in vacuum using a freeze-dryer (Bench Top Shell Bath Freezeer, VIRTIS, England). After freeze-drying, the kimchi was pulverized with a blender (KRUPS, Germany). The powder was kept under sterile conditions in a plastic bag at $-72^{\circ} \mathrm{C}$ until further used.

\section{Preparation of solid-state model media}

The fermentation medium used in this study was composed to simulate the substantial conditions of meat mixtures employed for the sausage production. The medium samples were prepared in $100 \mathrm{~mL}$ beakers. The ingredients are listed in Table 2. The start conditions of the model media were adjusted by the addition of corresponding salt concentrations as well as by setting appropriate $\mathrm{pH}$ values (Hechelmann, 1985; Koch, 1982; Liepe, 1985). The pH value was set with $0.5 \mathrm{~N} \mathrm{HCl}$ to 5.8 and then the medium was autoclaved for $20 \mathrm{~min}$ at $121^{\circ} \mathrm{C}$ and $1.2 \mathrm{bar}$. To avoid maillard reactions owing to heat treatment, glucose was sterilized separately and added aseptically to the medium after cooling. As a gelling agent for a fixed phase ("solid phase") agar was added to the fermentation broth in a concentration of $3.5 \mathrm{~g}$ agar/200 $\mathrm{mL}$ before autoclaving.

\section{Fermentation after treatment with kimchi or kim- chi-powder}

Fifty $\mathrm{mL}$ solid-state medium was prepared in a $100 \mathrm{~mL}$

Table 2. Composition of model-medium

\begin{tabular}{lc}
\hline \hline Materials and ingredients & Concentration $(\mathrm{g} / \mathrm{L})$ \\
\hline Meat extracts & 12.0 \\
Glucose & 10.0 \\
$\mathrm{NaCl}$ & 20.0 \\
Dipotassium hydrophosphate & 2.0 \\
$\mathrm{MgSO}_{4} \cdot 7 \mathrm{H}_{2} \mathrm{O}$ & 0.15 \\
Glutamate & 0.5 \\
Agar & 17.5 \\
\hline
\end{tabular}


beaker. To avoid the influence of sampling on the fermentation characteristics of kimchi under solid-state, 77 beakers in total (7 batches with 11 samples each) were prepared. Prepared media were cooled to $40^{\circ} \mathrm{C}$ after autoclaving. Prior to coagulation, kimchi $(0.5,1.0,1.5,3.0$, and $5.0 \%)$ and kimchi-powder $(0.2$ and $0.5 \%)$ were added. For cooling further and to solidify the treated medium was left at room temperature for $40 \mathrm{~min}$. Then the agar block was smashed with a spatula and cut into small cubes of approximately $0.5 \mathrm{~cm}$ in diameter. The samples were incubated at $25^{\circ} \mathrm{C}$ for $5 \mathrm{~d}$.

\section{Biochemical characterization of fermentation with kimchi and kimchi-powder}

The sampling of all experiments were carried out in duplicate after $0,4,8,12,16,24,36,48$ and then every $24 \mathrm{~h}$ during the fermentation period of $120 \mathrm{~h}$. Ten $\mathrm{g}$ of each sample were homogenized in $90 \mathrm{~mL}$ sterile physiological saline solution $(0.9 \% \mathrm{NaCl})$ for 2 min with a stomacher (Masticator, iUL, Spain). After sampling $1 \mathrm{~mL}$ from this suspension for the determination of CFU, the residue was filtered using a strainer and used to investigate $\mathrm{pH}$ and titratable acidity. The determination of the microbiological changes were investigated by the numbers of total viable counts (TVC) using Plate Count agar and lactic acid bacteria using MRS agar.

\section{Results and Discussion}

\section{Kimchi in the solid-state fermentation}

To evaluate the possibilities of alternative use of kimchi microorganisms as a substitute for the commercial starter cultures applied in sausage production, a series of microbiological and chemical experiments were carried out. The adaptation of LAB from kimchi to the special sausage conditions was investigated by evaluating their growth and ability to utilize fermenting sugar resulting in production of acids and lowering $\mathrm{pH}$. However, such investigations are very difficult to be surveyed in the real sausage because of an inevitable contamination with unidentified microorganisms deriving from the raw materials or the environment (Liepe et al., 1989). Furthermore, it is also very difficult to obtain reproducible and constant fermentation conditions in sausages (Katsaras and Leistner, 1988). Therefore, experiments were carried out using solid-state model media in which substantial conditions of a meat mixture prepared for sausage production were devised. The results are compared with those of previous trials carried out in submerged model medium (Lee and
Kunz, 2009) to investigate the effect of fixed phase on the microbiological and chemical characteristics of kimchi LAB.

The tendency of microbial changes in both TVC and LAB counts in the solid-state model medium was shown in Figs. 1 and 2. The initial TVC (Fig. 1) ranged from 6.95 Log CFU/g to $7.49 \mathrm{Log}$ CFU/g depending on the concentration of added kimchi. The highest TVCs reached around $8.90 \mathrm{Log}$ CFU/g in 12-24 h comparable with that in the submerged medium (around $9 \mathrm{Log} \mathrm{CFU} / \mathrm{mL}$ ) (Lee and Kunz, 2009). The initial TVC as well as the TVC during the fermentation period in each sample were closed to the counts of lactobacilli.

The initial LAB counts (Fig. 2) varied widely between 7.18 Log CFU/g and 8.34 Log CFU/g depending on the concentration of added kimchi. Smith and Palumbo (1983) suggested that an addition of large numbers (7-9 Log $\mathrm{CFU} / \mathrm{g}$ ) of desirable microorganisms would inhibit the growth of undesirable species, thereby preventing or

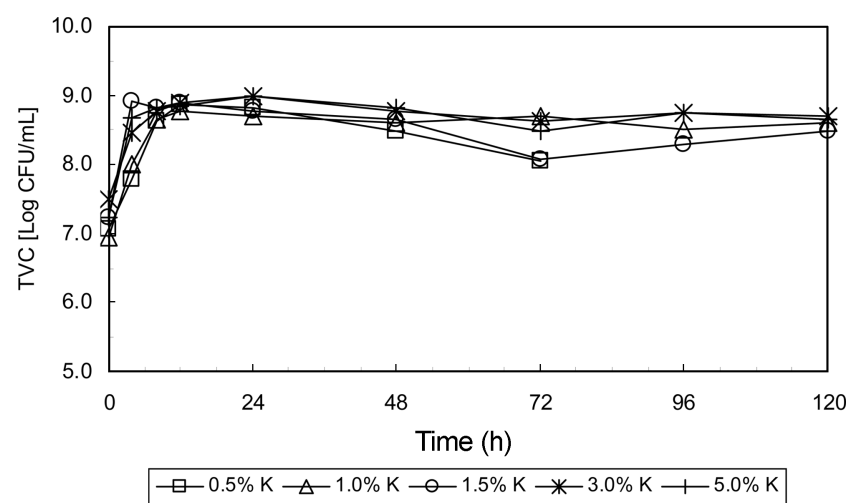

Fig. 1. Changes in total viable count introduced by the addition of kimchi at different concentrations (w/v) into the solid-state model medium as a function of fermentation time $(\mathrm{K}=$ kimchi $)$.

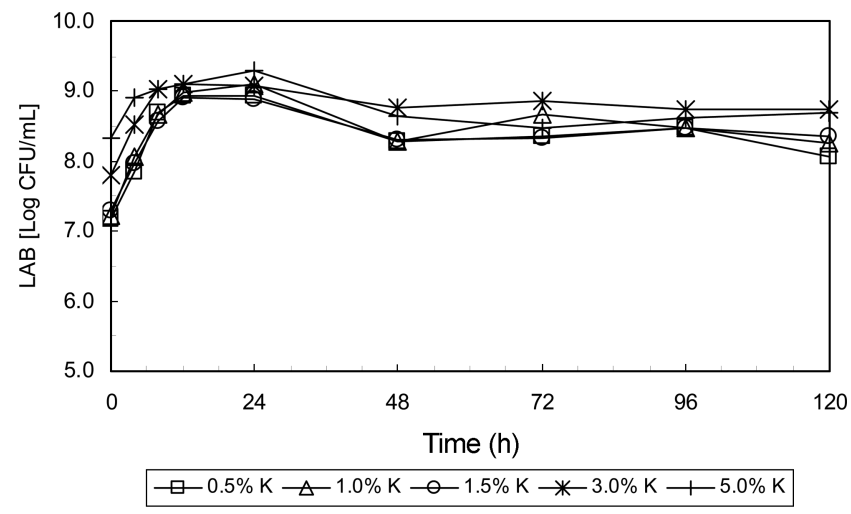

Fig. 2. Growth of lactic acid bacteria introduced by the addition of kimchi at different concentrations into the immobilized model medium as a function of fermentation time. 
reducing meat fermentation failures. After that, Luecke and Hechelmann (1985) stressed the importance of increasing the inoculation counts of LAB, contributing to the competitiveness against 'spontaneous microflora' and development of optimal sensory characteristics with more predictable and more rapid $\mathrm{pH}$ decrease. In the present work, the use of kimchi as an additive for the production of fermented sausages was resulted in the accompanying inoculums of LAB in the concentrations of more than 7 Log CFU/g.

An organism usually needs time to adapt to the new environment when inoculated, so called lag phase, in which it needs the time for the synthesis of enzymes to utilize the nutrients available (Garbutt, 1997). Such an adaptation time of LAB under the sausage condition is not needed by the use of kimchi under the solid-state fermentation. LAB population inoculated into the model medium had no lag phase but showed a logarithmical growth from the beginning of the fermentation.

Under the primary solid-state fermentation, as mentioned before, the nutrients are distributed almost homogeneously in mixed media. In a solid-state mixture, bacteria start to consume nutrients from their surroundings. As the fermentation progress, continues a depletion of nutrients in their surroundings. As the fermentation progress, continues a depletion of nutrients in their surroundings develops. In such a condition, the nutrients are supplied to the bacteria via diffusion. As a result, diffusion is a decisive factor in the determination of fermentation velocity. A slower metabolism and growth of bacteria caused by such conditions due to the solid-state fermentation was already observed in the works of Katsaras and Leistner (1988), Liepe (1987), and Liepe et al. (1989). In the work of Liepe (1987), for example, the numbers of viable bacterial counts in the immobilized condition were reduced for about 1 Log unit as compared to the liquid medium. According to the experimental results of the present work, the adaptation and growth of kimchi LAB was not influenced by the immobilized condition by showing no lag phase and thus acted similar as in the submerged medium (Lee and Kunz, 2009). The initially increased LAB counts reached the higher values of 8.93-9.11 Log $\mathrm{CFU} / \mathrm{mL}$ in $12 \mathrm{~h}$. These counts were comparable with or even higher than those in submerged medium (8.85-8.99 $\log \mathrm{CFU} / \mathrm{mL}$ ) although a decrease in the number of the LAB was observed after $24 \mathrm{~h}$ reaching below 9 Log CFU/mL (Lee and Kunz, 2009). After that, however, the counts were nearly maintained until the end of the fermentation period reaching final values of 8.08-8.70 Log
$\mathrm{CFU} / \mathrm{mL}$. This indicates that neither the mobility of the LAB nor the supply of fermenting sugar was limited by the immobilization. Such a result could be contributed to the enriched water by kimchi addition since the water content of kimchi is more than 80\% (Hwang, 1991; Park et al., 1996). This water from kimchi might have been located in the space between the particles ("cavities") and may have acted as a means of transport of the LAB and also of the nutrients. Such an effect provoked by the kimchi addition should be also expected in the real sausage system and it might negatively influence the sensorial development and hygienic stability (Leistner, 1985). It must be taken into account that the kimchi addition was also accompanied by an enrichment of the mixture with salt (3.0\% in kimchi). Kastaras and Leistner (1998) observed that any additional salt have penetrated the inside of medium particles and caused a swelling of particle owing to an absorption of nearby water. In this process, the molecules of the medium have been released from the inside of the medium particles to surroundings and supplied to the LAB. This effect could have positively influenced on the metabolism and growth of the LAB also in the present work.

Figs. 3 and 4 show the evolution of $\mathrm{pH}$ and lactic acid of the solid-state model medium treated with different concentrations of kimchi during the fermentation period of $120 \mathrm{~h}$. Although the initial $\mathrm{pH}$ values varied from 5.54 to 6.06 depending on the added amount of kimchi, a remarkable decrease in $\mathrm{pH}$ was observed in all batches during the initial $24 \mathrm{~h}$. The immobilized condition did not affect the growth of LAB from kimchi in the environment of fermented sausage, however it did affect the metabolism of LAB showing the longer time for reaching the lowest $\mathrm{pH}$ value and higher $\mathrm{pH}$ value than the submerged condition (Lee and Kunz, 2009). This tendency could be

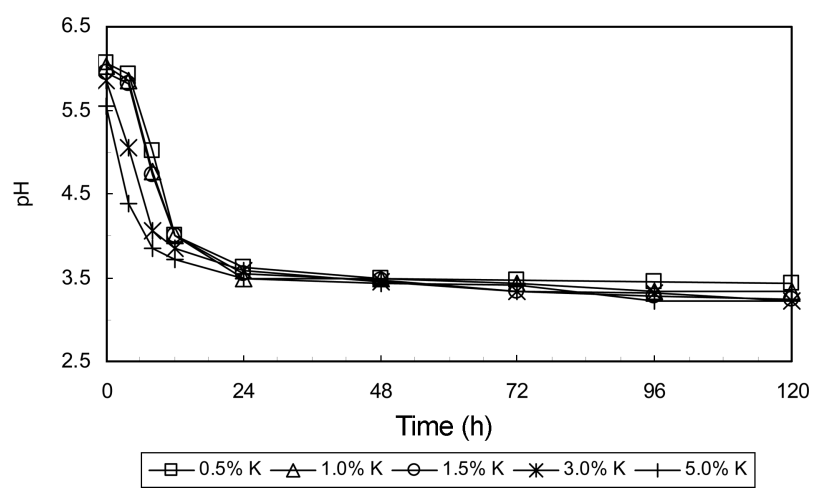

Fig. 3. Changes in pH of kimchi/medium-mixture under the immobilized condition as a function of fermentation time at different concentrations of added kimchi. 


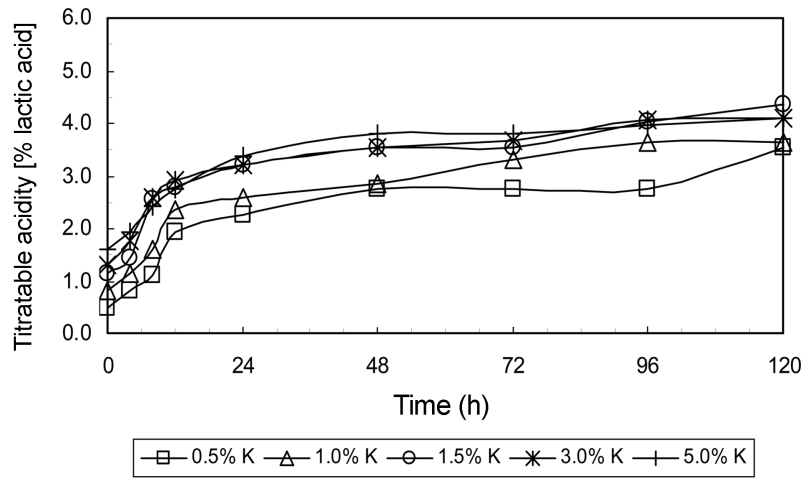

Fig. 4. Changes in lactic acid contents in the kimchi/mediummixture under the immobilized condition as a function of fermentation time at different concentrations of added kimchi.

also found in the evolution of lactic acid contents. The solid-state medium had the effect to lower the production of lactic acid by showing reduced lactic acid contents as compared with those in the submerged medium throughout the fermentation period (Fig. 4) (Lee and Kunz, 2009). After the $\mathrm{pH}$ values of the kimchi samples under the immobilized condition reached the deepest points, they underwent very little variation and reached final values of 3.43-3.22.

\section{Kimchi-powder in the submerged fermentation}

The microbial and physiological activities of the LAB in kimchi-powder were investigated using solid-state model media in the same manner used for kimchi LAB (Figs. 5 and 6). Even though the utility of kimchi LAB as a substitute for commercial starters in the production of sausages is advantageous due to their vital condition (Crueger, 1984), kimchi can present a handicap in its use due to its high water content. For example, an addition of $10 \%$ kimchi to the sausage mixture causes a moisture addition of approximately $8 \%$ of the sausage weight. For this reason, a dried form of kimchi (kimchi-powder) by using lyophilization was chosen to be studied for the properties of its $\mathrm{LAB}$ as starter culture for the production of fermented sausages. The lyophilized materials almost maintain their original volume, shape, aroma, color, enzyme and different kind of nutrients, and can be maintained for a long time (Jennings, 1997). Furthermore, lyophilization is regarded as the best method for preserving bacterial cultures. The metabolism of bacteria is interrupted by lyophilization but they can multiply again even after some years of storage (Chemie Lexikon, 1995). Therefore, the utility of freeze-dried kimchi-powder might compensate the disadvantageous features of kimchi but

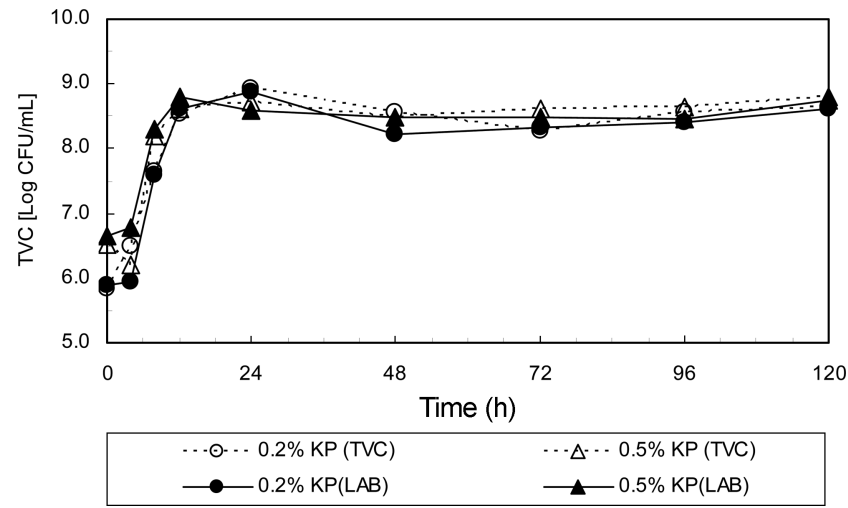

Fig. 5. Changes in total viable count (TVC) and lactic acid bacteria (LAB) count introduced by the addition of kimchi-powder (KP) at different concentrations into the immbolizied model medium as a function of fermentation time.

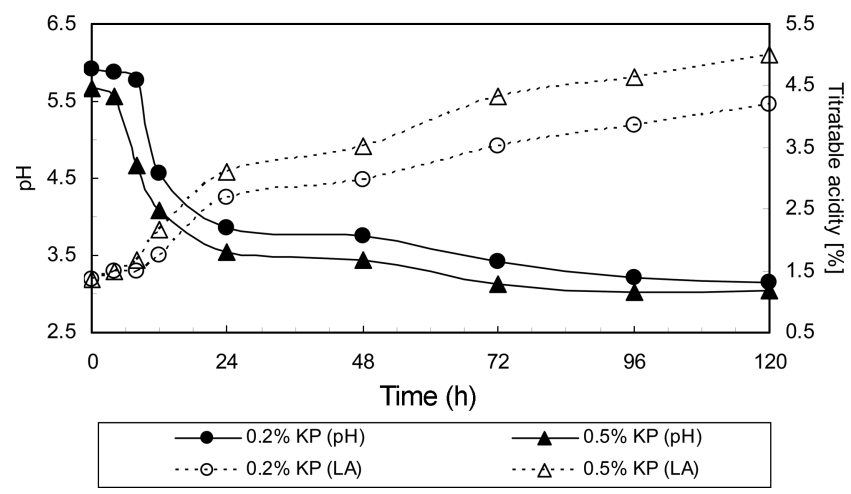

Fig. 6. Changes in $\mathrm{pH}$ and lactic acid contents in the kimchipowder/medium-mixture under the immobilized condition as a function of fermentation time at different concentrations of added kimchi-powder (LA=lactic acid).

with comparable microbiological and physiological values.

The changes in TVC and LAB counts introduced by the addition of kimchi-powder during fermentation are presented in Fig. 5. The TVCs initially increased logarithmically with the exception of the $0.5 \% \mathrm{KP}$ that had a decrease in the first $4 \mathrm{~h}$. The counts reached $8.53 \mathrm{log}$ $\mathrm{CFU} / \mathrm{mL}(0.2 \% \mathrm{KP})$ and $8.61 \log \mathrm{CFU} / \mathrm{mL}(0.5 \% \mathrm{KP})$ in $12 \mathrm{~h}$. After that the same values were remained more or less up to the end.

The values of TVC were similar to those of the LAB count over the fermentation period. An initial lag phase was observed in the kimchi-powder batches as shown in the submerged model medium (Lee and Kunz, 2009). This adaptation phase of LAB from kimchi-powder may due to the time needed for rehydration since they subsisted as lyophilized culture in freeze-dried form (Luecke 
and Hechelmann, 1986). As the metabolism and enzyme functions of the LAB in lyophilized kimchi-powder were at a standstill, and therefore the adaptation phase in the present work may have been necessary. Such a standstill of biological activity and chemical reactions can be also found in the initial period of lactic acid production and $\mathrm{pH}$ changes in the present work (Fig. 6).

The difference in the numbers of LAB counts between the batches ( $2 \% \mathrm{KP}$ and $5 \% \mathrm{KP})$ was continued during the growth phase, but after they entered into the stationary phase the difference began to narrow. The highest counts were $8.88 \mathrm{Log} \mathrm{CFU} / \mathrm{mL}(0.2 \% \mathrm{KP})$ and $8.58 \mathrm{Log}$ $\mathrm{CFU} / \mathrm{mL}(0.5 \% \mathrm{KP})$ after $24 \mathrm{~h}$ and these values remained relatively constant until the end of the investigation period. The values in this period (8.23-8.88) were similar to those of the kimchi-powder batches under the submerged condition (8.32-8.96) (Lee and Kunz, 2009). Although there was not much difference in their counts, the viable cell numbers in the solid-state batch treated with kimchi-powder were lower than those in the liquid medium. This is due to the fact that in solid-state condition the content of freely available water is very low as compared to the liquid medium (Katsaras and Leistner, 1988). In the case of kimchi-powder, the rehydration is an essential process for the recovery of its biological growth and chemical reaction (Luecke and Hechelmann, 1986). In the solid-state fermentation, this process may have been retarded or limited as compared to the submerged fermentation. Since the kimchi-powder was added into the solid-state model medium without any water treatment, the microorganisms in the eutectic mixture had to rely on the moisture that existed in cracks as well as on the surface of the medium particles for their rehydration (Luecke and Hechelmann, 1986). Accordingly, the water in the cracks and on the surface of the medium particles must have been absorbed by the kimchi-powder resulting in an enhanced drying of the mixture. As this should have slowed down the metabolic activity in the kimchi-powder batches, the reduced viable cell counts in the solid-state medium can be explained. However, the difference of two media was not too much with 0.08-0.09 Log CFU/mL.

The souring properties of the LAB have also been influenced by the consistency of the medium as both solidstate batches showed higher $\mathrm{pH}$ values and lower lactic acid productions throughout the fermentation than the liquid medium batches. The decrease of $\mathrm{pH}$ in kimchi-powder batches under the solid-state condition was slow during the initial 4-8 h, being consistent with a slow increase in the LAB counts. After an initially stationary phase of $\mathrm{pH}$, a sharp decrease followed up to $24 \mathrm{~h}$ and the value decreased slightly until the end of the fermentation. Final values were $3.14(0.2 \%)$ and $3.04(0.5 \%)$ and thus were slightly higher than the values in the submerged model medium (2.94 and 2.83, respectively) (Lee and Kunz, 2009). This coincided with a lower formation of lactic acid with final values of $4.19-5.00 \%$ in the solid-state model medium as compared with those in the submerged model medium of $4.65-5.55 \%$. Nevertheless, the $\mathrm{pH}$ drop in the solid-state batches was quite rapid and reached low values $(<4.0)$, demonstrating the excellent souring properties of kimchi-powder LAB. Furthermore, the slow-down of $\mathrm{pH}$ values of kimchi-powder samples in the solid-state media could contribute to a satisfactory aroma formation which can be impaired by preventing the growth of important aroma-forming microorganisms or by interfering with their nitrate reducing activities with the rapid reduction of $\mathrm{pH}$ (Roedel and stiebing, 1988).

In conclusion, the LAB integrated by the addition of kimchi or kimchi-powder under the solid-state model medium were able to fulfill the basic criteria for fermented sausage starter cultures by showing salt tolerance, good fermentation of sugar and metabolic activities at $25^{\circ} \mathrm{C}$ (Buckenhueskes, 1993; Hammes et al., 1985; Luecke and Hechelmann, 1985). In particular, the fermentation of kimchi-powder LAB under the real condition of sausages (solid-state immobilized condition) may make up the weak points of too much production of lactic acid and rapid $\mathrm{pH}$ reduction.

\section{Acknowledgments}

This study was supported by the Friedrich-Ebert-Stiftung, Germany with a grant for Joo-Yeon Lee. The study was carried out at Department of Food Technology, University of Bonn in Germany.

\section{References}

1. Bacus, J. N. and Brown, W. L. (1981) Use of microbial cultures: Meat Products. Food Tech. 74-78, 83.

2. Buckenhueskes, H. J. (1993) Selection criteria for lactic acid bacteria to be used as starter culture for various food commodities. FEMS 12, 253-272.

3. Chemie Lexikon (1995) - Version 1.0. Stuttgart/New York. Georg Thieme Verlag.

4. Crueger. (1984) Fermentationtechnik. In: Biotechnologiem, Lehrbuch der angewandten Mikrobiologie, 2. Auflage. Oldenbourg-Verlag, pp. 50-59.

5. Garbutt, J. (1997) Essentials of Food Microbiology. Arnold, 
London, pp. 41.

6. Geisen, R., Luecke, F. K., and Kroeckel, L. (1992) Starter and protective cultures for meat and meat products. Fleischwirtschaft 72, 894-898.

7. Jennings, T. A. (1997) Effect of formulation on lyophilization, part 1. IVD Techhnol. Jan. - Feb., 38-41.

8. Hammes, W. P. and Knauf, H. J. (1994) Starters in the processing of meat products. Meat Sci. 36, 155-168.

9. Hammes, W. P., Roelz, I., and Bantleon, A. (1985) Microbiologische Untersuchung der auf dem deutschen Markt vorhandenen Starterkulturepraeparate fuer die Rohwurstbereitung Fleischwirtschaft 65, 629-632, 635-636, 729-734.

10. Hechelmann, H. (1985) Microbiell verursachte Fehlfabrikate bei Rohwurst und Rohschinken. In: Mikrobiologie und Qualitaet von Rohwurst und Rohschinken. Kulmbacher Reihe Band 5. Kulmbach, pp.103-127.

11. Hwang, I. J. (1991) Ernaehrungsphysiologische Wirkung laktofermentierter Kohlgemuese-Einfluss regelmaessiger Aufnahme von Sauerkraut und Kimchi auf verschiedene Blutparameter. Ph. D. Thesis. Giessen.

12. Hwang, K. C., Chung, Y. S., and Kim, H. S. (1960) Microbiological studies on kimchi. II. Isolation and identification of aerobic bacteria. Bull. Sci. Res. Inst. 5, 51.

13. Kastaras, K. and Leistner, L. (1988) Topographie der Bakterien in der Rohwurst. Fleischwirtschaft 68, 1295-1298.

14. Koch, O. J. (1982) Die Fabrikation feiner Fleischund Wurstwaren. 17. Auflage. Franfurt, pp. 171-191.

15. Kroeckel, L (1995) Starterkulturen fuer die Rohwurst. AIDVerbraucherdienst 40, Heft 7, 147-154.

16. Kunz, B. (1989) Aspects in the use of starter cultures in meat products. ICoMST-89, 176-180.

17. Kunz, B. (1994) Grundriss der Lebensmittel-Mikrobiologie. 2. Ueberarbeitete und erweiterte Auflage. Hamburg: Behr's Verlag.

18. Lee, J. Y., Kim, C. J., and Kunz, B. (2006) Identification of lactic acid bacteria isolated from kimchi and studies on their suitability for application as starter culture in the production of fermented sausages. Meat Sci. 72, 437-445.
19. Lee, J. Y. and Kunz, B. (2009) Investigation of the Microbiological and Biochemical Properties of Kimchi in the Submerged Model System Designed for Fermented Sausages. Korean J. Food Sci. Ani. Resour. 29, 423-429.

20. Leistner, L. (1985) Allgemeins ueber Rohwurst und rohschinken. In: Microbiological und Qualitaet von Rohwurst und Rohschinken. Kulmbacher reih Band 5, Kulmbach: Institut fuer Mikrobiologie, Toxikologie und Histologie der Bundesanstalt fuer Fleischforschung, pp. 1-29.

21. Liepe, H. U. (1987) Keimverteilung in einer Rohwurst. Fleischwirtschaft 67, 1173-1176.

22. Liepe, H. U. (1985) Einsatz einer neuen Laktobazillen-Starterkultur fuer die Rohwurst-Technologie. Fleischwirtschaft 65, 1246-1247.

23. Liepe, H. J., Pfeil, E., and Porobi, R. (1989) Einfluss von Zuckerstoffen und Bakterien auf den Verlauf der Rohwurstsaeuerung. Fleischwirtschaft 69, 1173-1176.

24. Luecke, F. -K. and Hechelmann, H. (1985) Starterkulturen fuer Rohwurst und Rohschinken. In: Microbiological und Qualitaet von Rohwurst und Rohschinken. Kulmbacher Reihe Band 5, Kulmbach: Institut fuer Mmikrobiologie, Toxikologie und Histologie der Bundesanstalt fuer Fleischforschung, pp. 193-218.

25. Luecke, F. -K. and Hechelmann, H. (1986) Starterkulturen fuer Rohwurst und Rohschinken. Fleischwirtschaft 66, 154166.

26. Park, K. Y., Ha, J. O., and Rhee, S. H. (1996) A study on the control of dietary fibers and crude fiber in kimchi ingredients and kimchi. J. Korean Food Sci. Nutr. 25, 69-75.

27. Roca, M. and Incze, K. (1990) Fermented sausages. Food Rev. International 6, 91-118.

28. Roedel, W. and Stiebing, A. (1988) Continuous measurement of the ripening pattern in dry sausage. Fleischwirtschaft $\mathbf{6 8}$, 1423-1426.

29. Smith, J. L. and Palumbo, S. A. (1983) Use of starter cultures in meats. J. Food Prot. 46, 997-1006.

(Received 2010.3.9/Revised 2010.4.14/Accepted 2010.4.16) 